\title{
A VOLUME COMPARISON THEOREM FOR ASYMPTOTICALLY HYPERBOLIC MANIFOLDS
}

\author{
SIMON BRENDLE AND OTIS CHODOSH
}

\begin{abstract}
We define a notion of renormalized volume of an asymptotically hyperbolic manifold. Moreover, we prove a sharp volume comparison theorem for metrics with scalar curvature at least -6 . Finally, we show that the inequality is strict unless the metric is isometric to one of the Anti-deSitter-Schwarzschild metrics.
\end{abstract}

\section{INTRODUCTION}

Let $(\bar{M}, \bar{g})$ denote the standard three-dimensional hyperbolic space, so that

$$
\bar{g}=\frac{1}{1+s^{2}} d s \otimes d s+s^{2} g_{S^{2}}
$$

Let us consider a Riemannian metric $g$ on $M=\bar{M} \backslash K$, where $K$ is a bounded domain with smooth connected boundary. We assume that $g$ is asymptotically hyperbolic in the sense that $|g-\bar{g}|_{\bar{g}}=O\left(s^{-2-4 \delta}\right)$ for some $\delta \in\left(0, \frac{1}{4}\right)$ and $|\bar{D}(g-\bar{g})|_{\bar{g}}=o(1)$. We define the renormalized volume of $(M, g)$ by

$$
V(M, g):=\lim _{i \rightarrow \infty}\left(\operatorname{vol}\left(\Omega_{i} \cap M, g\right)-\operatorname{vol}\left(\Omega_{i}, \bar{g}\right)\right),
$$

where $\Omega_{i}$ is an arbitrary exhaustion of $\bar{M}$ by compact sets. The condition $|g-\bar{g}|_{\bar{g}}=O\left(s^{-2-4 \delta}\right)$ guarantees that the quantity $V(M, g)$ does not depend on the choice of the exhaustion $\Omega_{i}$. Clearly, $V(\bar{M}, \bar{g})=0$.

As an example, let us consider the Anti-deSitter-Schwarzschild manifold with mass $m>0$. To that end, let $s_{0}=s_{0}(m)$ denote the unique positive solution of the equation $1+s_{0}^{2}-m s_{0}^{-1}=0$. We then consider the manifold $\bar{M}_{m}=\bar{M} \backslash\left\{s \leq s_{0}(m)\right\}$ equipped with the Riemannian metric

$$
\bar{g}_{m}=\frac{1}{1+s^{2}-m s^{-1}} d s \otimes d s+s^{2} g_{S^{2}} .
$$

The boundary $S^{2} \times\left\{s_{0}(m)\right\}$ is an outermost minimal surface, which is referred to as the horizon. Moreover, it is easy to see that $\left|\bar{g}_{m}-\bar{g}\right|_{\bar{g}}=O\left(s^{-3}\right)$, so $\bar{g}$ satisfies the asymptotic assumptions above. The renormalized volume

The first author was supported in part by the National Science Foundation under grant DMS-1201924. The second author was supported in part by a National Science Foundation Graduate Research Fellowship DGE-1147470. 
of $\left(\bar{M}_{m}, \bar{g}_{m}\right)$ is given by

$$
V\left(\bar{M}_{m}, \bar{g}_{m}\right)=\lim _{r \rightarrow \infty}\left(\int_{s_{0}(m)}^{r} \frac{4 \pi s^{2}}{\sqrt{1+s^{2}-m s^{-1}}} d s-\int_{0}^{r} \frac{4 \pi s^{2}}{\sqrt{1+s^{2}}} d s\right) .
$$

We now state the main result of this paper.

Theorem 1. Let us consider a Riemannian metric $g$ on $M=\bar{M} \backslash K$, where $K$ is a compact set with smooth connected boundary. We assume that $g$ has the following properties:

- The manifold $(M, g)$ is asymptotically hyperbolic in the sense that $|g-\bar{g}|_{\bar{g}}=O\left(s^{-2-4 \delta}\right)$ and $|\bar{D}(g-\bar{g})|_{\bar{g}}=o(1)$.

- The scalar curvature of $g$ is at least -6 .

- The boundary $\partial M$ is an outermost minimal surface with respect to $g$, and we have area $(\partial M, g) \geq \operatorname{area}\left(\partial \bar{M}_{m}, \bar{g}_{m}\right)$ for some $m>0$.

Then $V(M, g) \geq V\left(\bar{M}, \bar{g}_{m}\right)$. Moreover, if equality holds, then $g$ is isometric to $\bar{g}_{m}$.

We note that our asymptotic assumptions are quite weak: in particular, $g$ and $\bar{g}_{m}$ may have different mass at infinity. An immediate consequence of Theorem 11 is that the function $m \mapsto V\left(\bar{M}_{m}, \bar{g}_{m}\right)$ is strictly monotone increasing. This fact is not obvious, as $s_{0}(m)$ is an increasing function of $m$.

Theorem 1 is motivated in part by Bray's volume comparison theorem [1] for three-manifolds with scalar curvature at least 6 , as well as by a rigidity result due to Llarull [9]. A survey of this and other rigidity results involving scalar curvature can be found in [4].

The proof of Theorem 1 uses two main ingredients. The first is the weak inverse mean curvature flow, which was introduced in the ground-breaking work of Huisken and Ilmanen [8] on the Riemannian Penrose inequality (see also [2], where an alternative proof is given). The inverse mean curvature flow has also been considered as a possible tool for proving a version of the Penrose inequality for asymptotically hyperbolic manifolds; see [10, [11. More recently, the inverse mean curvature flow was used in [6] to prove a sharp Minkowski-type inequality for surfaces in the Anti-deSitterSchwarzschild manifold.

The second ingredient in our argument is an isoperimetric principle which asserts that a coordinate sphere in the standard Anti-deSitter-Schwarzschild manifold has smallest area among all surfaces that are homologous to the horizon and enclose the same amount of volume. This inequality was established in [7] following earlier work by Bray [1. In fact, it is known that the coordinate spheres are the only embedded hypersurfaces with constant mean curvature in the Anti-deSitter-Schwarzschild manifold (see [5]).

Our approach also shares common features with a result of Bray and Miao [3], which gives a sharp bound for the capacity of a surface in a threemanifold of nonnegative scalar curvature. 


\section{Proof of Theorem 1}

Let $(M, g)$ be a Riemannian manifold which satisfies the assumptions of Theorem 1, and let $\left(\bar{M}_{m}, \bar{g}_{m}\right)$ be an Anti-deSitter-Schwarzschild manifold satisfying area $(\partial M, g) \geq \operatorname{area}\left(\partial \bar{M}_{m}, \bar{g}_{m}\right)$. For abbreviation, let $A=$ area $(\partial M, g)$ and $\bar{A}=\operatorname{area}\left(\partial \bar{M}_{m}, \bar{g}_{m}\right)$.

Let $\Sigma_{t}$ denote the weak solution of the inverse mean curvature flow in $(M, g)$ with the initial surface $\Sigma_{0}=\partial M$. For each $t$, we denote by $\Omega_{t} \subset \bar{M}$ the region bounded by $\Sigma_{t}$.

Proposition 2. Let $\delta \in\left(0, \frac{1}{4}\right)$ be as above. Then we have $\left\{s \leq e^{\frac{(1-\delta) t}{2}}\right\} \subset \Omega_{t}$ for $t$ sufficiently large.

Proof. The coordinate sphere $S^{2} \times\{s\}$ has mean curvature $2+o(1)$ for $s$ large. Hence, we can find a real number $t_{0}$ such that the surfaces $S_{t}=\left\{s=e^{\frac{(1-2 \delta) t}{2}}\right\}$ move with a speed less than $\frac{1}{H}$ for $t \geq t_{0}$. By the Weak Existence Theorem 3.1 in [8], the regions $\Omega_{t}$ will eventually contain every given compact set. Hence, we can find a real number $\tau$ such that $\left\{s \leq e^{\frac{(1-2 \delta) t_{0}}{2}}\right\} \subset \Omega_{\tau}$. By the maximum principle (cf. Theorem 2.2 in [8]), we have $\left\{s \leq e^{\frac{(1-2 \delta)\left(t-\tau+t_{0}\right)}{2}}\right\} \subset \Omega_{t}$ for $t \geq \tau$. From this, the assertion follows.

Since the boundary $\partial M$ is an outermost minimal surface, we have area $\left(\Sigma_{t}, g\right)=$ $e^{t} A$. Moreover, it is well known that the quantity

$$
m_{H}\left(\Sigma_{t}\right)=\operatorname{area}\left(\Sigma_{t}, g\right)^{\frac{1}{2}}\left(16 \pi-\int_{\Sigma_{t}}\left(H_{g}^{2}-4\right) d \mu_{g}\right)
$$

is monotone increasing in $t$.

Proposition 3. For each $\tau \geq 0$, we have

$$
\operatorname{vol}\left(\Omega_{\tau} \cap M, g\right) \geq \int_{0}^{\tau} e^{\frac{3 t}{2}} A^{\frac{3}{2}}\left(4 e^{t} A+16 \pi-e^{-\frac{t}{2}} A^{-\frac{1}{2}} m_{H}\left(\Sigma_{t}\right)\right)^{-\frac{1}{2}} d t .
$$

Proof. By the co-area formula, we have

$$
\int_{\Omega_{\tau} \cap M} \psi H_{g} d \operatorname{vol}_{g}=\int_{0}^{\tau}\left(\int_{\Sigma_{t}} \psi d \mu_{g}\right) d t
$$

for every nonnegative measurable function $\psi$. Hence, if we put

$$
\psi= \begin{cases}\frac{1}{H_{g}} & \text { if } H_{g}>0 \\ \infty & \text { if } H_{g}=0\end{cases}
$$

then we obtain

$$
\operatorname{vol}\left(\Omega_{\tau} \cap M, g\right) \geq \int_{0}^{\tau}\left(\int_{\Sigma_{t}} \psi d \mu_{g}\right) d t
$$


Moreover, it follows from Hölder's inequality that

$$
\begin{aligned}
\int_{\Sigma_{t}} \psi d \mu_{g} & \geq \operatorname{area}\left(\Sigma_{t}, g\right)^{\frac{3}{2}}\left(\int_{\Sigma_{t}} H_{g}^{2} d \mu_{g}\right)^{-\frac{1}{2}} \\
& =\operatorname{area}\left(\Sigma_{t}, g\right)^{\frac{3}{2}}\left(4 \operatorname{area}\left(\Sigma_{t}, g\right)+16 \pi-\operatorname{area}\left(\Sigma_{t}, g\right)^{-\frac{1}{2}} m_{H}\left(\Sigma_{t}\right)\right)^{-\frac{1}{2}} \\
& =e^{\frac{3 t}{2}} A^{\frac{3}{2}}\left(4 e^{t} A+16 \pi-e^{-\frac{t}{2}} A^{-\frac{1}{2}} m_{H}\left(\Sigma_{t}\right)\right)^{-\frac{1}{2}}
\end{aligned}
$$

Putting these facts together, the assertion follows.

Corollary 4. We have

$$
2 \operatorname{vol}\left(\Omega_{\tau} \cap M, g\right) \geq \int_{0}^{\tau} e^{t} A^{\frac{3}{2}}\left(\left(1-e^{-\frac{3 t}{2}}\right) A+4 \pi\left(e^{-t}-e^{-\frac{3 t}{2}}\right)\right)^{-\frac{1}{2}} d t .
$$

Proof. Using the monotonicity of $m_{H}\left(\Sigma_{t}\right)$, we obtain

$$
m_{H}\left(\Sigma_{t}\right) \geq m_{H}\left(\Sigma_{0}\right)=4 A^{\frac{1}{2}}(A+4 \pi) .
$$

This implies

$$
2 \operatorname{vol}\left(\Omega_{\tau} \cap M, g\right) \geq \int_{0}^{\tau} e^{\frac{3 t}{2}} A^{\frac{3}{2}}\left(\left(e^{t}-e^{-\frac{t}{2}}\right) A+4 \pi\left(1-e^{-\frac{t}{2}}\right)\right)^{-\frac{1}{2}} d t .
$$

From this, the assertion follows.

Proposition 5. Let $\Omega$ be a domain in $\bar{M}$ such that $\left\{s \leq s_{0}(m)\right\} \subset \Omega$, and let $\Sigma$ denote the boundary of $\Omega$. Then

$$
2 \operatorname{vol}\left(\Omega \cap \bar{M}_{m}, \bar{g}_{m}\right) \leq \int_{0}^{\bar{\tau}} e^{t} \bar{A}^{\frac{3}{2}}\left(\left(1-e^{-\frac{3 t}{2}}\right) \bar{A}+4 \pi\left(e^{-t}-e^{-\frac{3 t}{2}}\right)\right)^{-\frac{1}{2}} d t,
$$

where $\bar{\tau}$ is defined by area $\left(\Sigma, \bar{g}_{m}\right)=e^{\bar{\tau}} \bar{A}$.

Proof. If $\Sigma$ is a coordinate sphere in $\left(\bar{M}_{m}, \bar{g}_{m}\right)$, then we have

$$
2 \operatorname{vol}\left(\Omega \cap \bar{M}_{m}, \bar{g}_{m}\right)=\int_{0}^{\bar{\tau}} e^{t} \bar{A}^{\frac{3}{2}}\left(\left(1-e^{-\frac{3 t}{2}}\right) \bar{A}+4 \pi\left(e^{-t}-e^{-\frac{3 t}{2}}\right)\right)^{-\frac{1}{2}} d t,
$$

where $\bar{\tau}$ is defined by area $\left(\Sigma, \bar{g}_{m}\right)=e^{\bar{\tau}} \bar{A}$. On the other hand, it is known (cf. [7], Theorem 4.2) that the coordinate spheres in $\left(\bar{M}_{m}, \bar{g}_{m}\right)$ enclose the largest volume for any given surface area. Putting these facts together, the assertion follows.

Let us consider a sequence of times $\tau_{i} \rightarrow \infty$. Moreover, we define a sequence of times $\bar{\tau}_{i} \rightarrow \infty$ by area $\left(\Sigma_{\tau_{i}}, \bar{g}_{m}\right)=e^{\bar{\tau}_{i}} \bar{A}$. By Proposition 2, we have $s \geq e^{\frac{(1-\delta) t}{2}}$ on $\Sigma_{t}$ if $t$ is large enough. This implies

$$
\left|g-\bar{g}_{m}\right|_{\bar{g}_{m}} \leq O\left(s^{-2-4 \delta}\right) \leq O\left(e^{-(1-\delta)(1+2 \delta) t}\right)
$$


at each point on $\Sigma_{t}$. From this, we deduce that

$$
\begin{aligned}
e^{\tau_{i}} A & =\operatorname{area}\left(\Sigma_{\tau_{i}}, g\right) \\
& =\operatorname{area}\left(\Sigma_{\tau_{i}}, \bar{g}_{m}\right)\left(1+O\left(e^{-(1-\delta)(1+2 \delta) \tau_{i}}\right)\right) \\
& =e^{\bar{\tau}_{i}} \bar{A}\left(1+O\left(e^{-(1-\delta)(1+2 \delta) \tau_{i}}\right)\right) .
\end{aligned}
$$

Thus, we conclude that

$$
\tau_{i}=\bar{\tau}_{i}-\alpha+O\left(e^{-(1-\delta)(1+2 \delta) \tau_{i}}\right),
$$

where $\alpha=\log (A / \bar{A}) \geq 0$. Note that $(1-\delta)(1+2 \delta)>1$ since $\delta \in\left(0, \frac{1}{4}\right)$.

By Corollary 4, we have

$$
\begin{aligned}
2 \operatorname{vol}\left(\Omega_{\tau_{i}} \cap M, g\right) & \geq \int_{0}^{\tau_{i}} e^{t} A^{\frac{3}{2}}\left(\left(1-e^{-\frac{3 t}{2}}\right) A+4 \pi\left(e^{-t}-e^{-\frac{3 t}{2}}\right)\right)^{-\frac{1}{2}} d t \\
& =\int_{\alpha}^{\tau_{i}+\alpha} e^{t-\alpha} A^{\frac{3}{2}}\left(\left(1-e^{-\frac{3 t-3 \alpha}{2}}\right) A+4 \pi\left(e^{-t+\alpha}-e^{-\frac{3 t-3 \alpha}{2}}\right)\right)^{-\frac{1}{2}} d t \\
& =\int_{\alpha}^{\tau_{i}+\alpha} e^{t} \bar{A}^{\frac{3}{2}}\left(\left(1-e^{-\frac{3 t-3 \alpha}{2}}\right) \bar{A}+4 \pi\left(e^{-t}-e^{-\frac{3 t-\alpha}{2}}\right)\right)^{-\frac{1}{2}} d t .
\end{aligned}
$$

On the other hand, we have

$$
2 \operatorname{vol}\left(\Omega_{\tau_{i}} \cap \bar{M}_{m}, \bar{g}_{m}\right) \leq \int_{0}^{\bar{\tau}_{i}} e^{t} \bar{A}^{\frac{3}{2}}\left(\left(1-e^{-\frac{3 t}{2}}\right) \bar{A}+4 \pi\left(e^{-t}-e^{-\frac{3 t}{2}}\right)\right)^{-\frac{1}{2}} d t
$$

by Proposition 5. Putting these facts together, we obtain

$$
\begin{aligned}
& 2\left(V(M, g)-V\left(\bar{M}_{m}, \bar{g}_{m}\right)\right) \\
& =\limsup _{i \rightarrow \infty} 2\left(\operatorname{vol}\left(\Omega_{\tau_{i}} \cap M, g\right)-\operatorname{vol}\left(\Omega_{\tau_{i}} \cap \bar{M}_{m}, \bar{g}_{m}\right)\right) \\
& \geq \limsup _{i \rightarrow \infty}\left(\int_{\alpha}^{\tau_{i}+\alpha} e^{t} \bar{A}^{\frac{3}{2}}\left(\left(1-e^{-\frac{3 t-3 \alpha}{2}}\right) \bar{A}+4 \pi\left(e^{-t}-e^{-\frac{3 t-\alpha}{2}}\right)\right)^{-\frac{1}{2}} d t\right. \\
& \left.-\int_{0}^{\bar{\tau}_{i}} e^{t} \bar{A}^{\frac{3}{2}}\left(\left(1-e^{-\frac{3 t}{2}}\right) \bar{A}+4 \pi\left(e^{-t}-e^{-\frac{3 t}{2}}\right)\right)^{-\frac{1}{2}} d t\right) \\
& =\limsup _{i \rightarrow \infty}\left(\int_{\alpha}^{\bar{\tau}_{i}} e^{t} \bar{A}^{\frac{3}{2}}\left(\left(1-e^{-\frac{3 t-3 \alpha}{2}}\right) \bar{A}+4 \pi\left(e^{-t}-e^{-\frac{3 t-\alpha}{2}}\right)\right)^{-\frac{1}{2}} d t\right. \\
& \left.-\int_{0}^{\bar{\tau}_{i}} e^{t} \bar{A}^{\frac{3}{2}}\left(\left(1-e^{-\frac{3 t}{2}}\right) \bar{A}+4 \pi\left(e^{-t}-e^{-\frac{3 t}{2}}\right)\right)^{-\frac{1}{2}} d t\right) \\
& =\bar{A}^{\frac{3}{2}} I(\alpha),
\end{aligned}
$$

where

$$
\begin{gathered}
I(\alpha)=\int_{\alpha}^{\infty} e^{t}\left[\left(\left(1-e^{-\frac{3 t-3 \alpha}{2}}\right) \bar{A}+4 \pi\left(e^{-t}-e^{-\frac{3 t-\alpha}{2}}\right)\right)^{-\frac{1}{2}}\right. \\
\left.-\left(\left(1-e^{-\frac{3 t}{2}}\right) \bar{A}+4 \pi\left(e^{-t}-e^{-\frac{3 t}{2}}\right)\right)^{-\frac{1}{2}}\right] d t \\
-\int_{0}^{\alpha} e^{t}\left(\left(1-e^{-\frac{3 t}{2}}\right) \bar{A}+4 \pi\left(e^{-t}-e^{-\frac{3 t}{2}}\right)\right)^{-\frac{1}{2}} d t .
\end{gathered}
$$


It is shown in the appendix that the function $I(\alpha)$ is positive for all $\alpha>0$. Thus, we conclude that $V(M, g) \geq V\left(\bar{M}_{m}, \bar{g}_{m}\right)$.

Finally, we analyze the case of equality. Suppose that $V(M, g)=V\left(\bar{M}_{m}, \bar{g}_{m}\right)$. Then $I(\alpha) \leq 0$, which implies that $\alpha=0$. Moreover, the difference

$$
2 \operatorname{vol}\left(\Omega_{\tau_{i}} \cap M, g\right)-\int_{0}^{\tau_{i}} e^{t} A^{\frac{3}{2}}\left(\left(1-e^{-\frac{3 t}{2}}\right) A+4 \pi\left(e^{-t}-e^{-\frac{3 t}{2}}\right)\right)^{-\frac{1}{2}} d t
$$

must converge to 0 as $i \rightarrow \infty$. Using Proposition [3, we conclude that $m_{H}\left(\Sigma_{t}\right)=m_{H}\left(\Sigma_{0}\right)$ for all $t$. This implies that $g$ is the isometric to one of the standard Anti-deSitter-Schwarzschild metrics. Since $\alpha=0$, the manifolds $(M, g)$ and $\left(\bar{M}_{m}, \bar{g}_{m}\right)$ have the same boundary area. Therefore, they are isometric.

\section{Appendix A. Positivity of the function $I(\alpha)$}

In this section, we show that $I(\alpha)>0$ for all $\alpha>0$. We begin with a lemma:

Lemma 6. Let $\varepsilon$ and $\mu$ be positive real numbers. If the ratio $\frac{\varepsilon}{\mu}$ is sufficiently small, then we have

$$
3 \mu \int_{0}^{\infty} e^{-\frac{t}{2}}\left(\varepsilon+\left(1-e^{-\frac{3 t}{2}}\right) \mu\right)^{-\frac{3}{2}} d t \geq 4 \varepsilon^{-\frac{1}{2}}+\mu^{-\frac{1}{2}} .
$$

Proof. It is elementary to check that

$$
e^{t} \geq 1+\frac{2}{3}\left(1-e^{-\frac{3 t}{2}}\right)
$$

hence

$$
e^{-\frac{t}{2}} \geq e^{-\frac{3 t}{2}}+\frac{2}{3} e^{-\frac{3 t}{2}}\left(1-e^{-\frac{3 t}{2}}\right)
$$

for all $t \geq 0$. This implies

$$
\begin{aligned}
& \int_{0}^{1} e^{-\frac{t}{2}}\left(\varepsilon+1-e^{-\frac{3 t}{2}}\right)^{-\frac{3}{2}} d t \\
& \geq \int_{0}^{1} e^{-\frac{3 t}{2}}\left(\varepsilon+1-e^{-\frac{3 t}{2}}\right)^{-\frac{3}{2}} d t+\frac{2}{3} \int_{0}^{1} e^{-\frac{3 t}{2}}\left(1-e^{-\frac{3 t}{2}}\right)\left(\varepsilon+1-e^{-\frac{3 t}{2}}\right)^{-\frac{3}{2}} d t \\
& =\int_{0}^{1} e^{-\frac{3 t}{2}}\left(\varepsilon+1-e^{-\frac{3 t}{2}}\right)^{-\frac{3}{2}} d t+\frac{2}{3} \int_{0}^{1} e^{-\frac{3 t}{2}}\left(1-e^{-\frac{3 t}{2}}\right)^{-\frac{1}{2}} d t-o(1) \\
& =\frac{4}{3} \varepsilon^{-\frac{1}{2}}-\frac{4}{3}\left(\varepsilon+1-e^{-\frac{3}{2}}\right)^{-\frac{1}{2}}+\frac{8}{9}\left(1-e^{-\frac{3}{2}}\right)^{\frac{1}{2}}-o(1)
\end{aligned}
$$


for $\varepsilon>0$ sufficiently small. Hence, we obtain

$$
\begin{aligned}
& \int_{0}^{\infty} e^{-\frac{t}{2}}\left(\varepsilon+1-e^{-\frac{3 t}{2}}\right)^{-\frac{3}{2}} d t \\
& \geq \frac{4}{3} \varepsilon^{-\frac{1}{2}}-\frac{4}{3}\left(\varepsilon+1-e^{-\frac{3}{2}}\right)^{-\frac{1}{2}}+\frac{8}{9}\left(1-e^{-\frac{3}{2}}\right)^{\frac{1}{2}}+(\varepsilon+1)^{-\frac{3}{2}} \int_{1}^{\infty} e^{-\frac{t}{2}} d t-o(1) \\
& \geq \frac{4}{3} \varepsilon^{-\frac{1}{2}}-\frac{4}{3}\left(1-e^{-\frac{3}{2}}\right)^{-\frac{1}{2}}+\frac{8}{9}\left(1-e^{-\frac{3}{2}}\right)^{\frac{1}{2}}+2 e^{-\frac{1}{2}}-o(1) \\
& \geq \frac{4}{3} \varepsilon^{-\frac{1}{2}}+\frac{1}{3}
\end{aligned}
$$

if $\varepsilon>0$ is small enough. This proves the assertion for $\mu=1$. The general case follows by scaling.

We now consider the function

$$
\begin{gathered}
I_{\varepsilon}(\alpha)=\int_{\alpha}^{\infty} e^{t}\left[\left(\varepsilon+\left(1-e^{-\frac{3 t-3 \alpha}{2}}\right) \bar{A}+4 \pi\left(e^{-t}-e^{-\frac{3 t-\alpha}{2}}\right)\right)^{-\frac{1}{2}}\right. \\
\left.-\left(\varepsilon+\left(1-e^{-\frac{3 t}{2}}\right) \bar{A}+4 \pi\left(e^{-t}-e^{-\frac{3 t}{2}}\right)\right)^{-\frac{1}{2}}\right] d t \\
-\int_{0}^{\alpha} e^{t}\left(\varepsilon+\left(1-e^{-\frac{3 t}{2}}\right) \bar{A}+4 \pi\left(e^{-t}-e^{-\frac{3 t}{2}}\right)\right)^{-\frac{1}{2}} d t .
\end{gathered}
$$

Then

$$
\begin{aligned}
\frac{d}{d \alpha} I_{\varepsilon}(\alpha)= & \int_{\alpha}^{\infty} e^{t} \frac{d}{d \alpha}\left[\left(\varepsilon+\left(1-e^{-\frac{3 t-3 \alpha}{2}}\right) \bar{A}+4 \pi\left(e^{-t}-e^{-\frac{3 t-\alpha}{2}}\right)\right)^{-\frac{1}{2}}\right] d t-e^{\alpha} \varepsilon^{-\frac{1}{2}} \\
= & \frac{1}{4}\left(3 e^{\frac{3 \alpha}{2}} \bar{A}+4 \pi e^{\frac{\alpha}{2}}\right) \\
& \cdot \int_{\alpha}^{\infty} e^{-\frac{t}{2}}\left(\varepsilon+\left(1-e^{-\frac{3 t-3 \alpha}{2}}\right) \bar{A}+4 \pi\left(e^{-t}-e^{-\frac{3 t-\alpha}{2}}\right)\right)^{-\frac{3}{2}} d t-e^{\alpha} \varepsilon^{-\frac{1}{2}} \\
= & \frac{e^{\alpha}}{4}\left(3 \bar{A}+4 \pi e^{-\alpha}\right) \\
& \cdot \int_{0}^{\infty} e^{-\frac{t}{2}}\left(\varepsilon+\left(1-e^{-\frac{3 t}{2}}\right) \bar{A}+4 \pi e^{-\alpha}\left(e^{-t}-e^{-\frac{3 t}{2}}\right)\right)^{-\frac{3}{2}} d t-e^{\alpha} \varepsilon^{-\frac{1}{2}} \\
\geq & \frac{e^{\alpha}}{4}\left(3 \bar{A}+4 \pi e^{-\alpha}\right) \\
& \cdot \int_{0}^{\infty} e^{-\frac{t}{2}}\left(\varepsilon+\left(1-e^{-\frac{3 t}{2}}\right)\left(\bar{A}+\frac{4 \pi}{3} e^{-\alpha}\right)\right)^{-\frac{3}{2}} d t-e^{\alpha} \varepsilon^{-\frac{1}{2}}
\end{aligned}
$$

where in the last step we have used the inequality $e^{-t}-e^{-\frac{3 t}{2}} \leq \frac{1}{3}\left(1-e^{-\frac{3 t}{2}}\right)$. Hence, if the ratio $\frac{\varepsilon}{\bar{A}+\frac{4 \pi}{3} e^{-\alpha}}$ is sufficiently small, then

$$
\frac{d}{d \alpha} I_{\varepsilon}(\alpha) \geq \frac{e^{\alpha}}{4}\left(\bar{A}+\frac{4 \pi}{3} e^{-\alpha}\right)^{-\frac{1}{2}}
$$

by Lemma 6. Since $I(\alpha)=\lim _{\varepsilon \rightarrow 0} I_{\varepsilon}(\alpha)$ for each $\alpha \geq 0$, we conclude that the function $I(\alpha)$ is strictly monotone increasing. In particular, $I(\alpha)>0$ for all $\alpha>0$. 


\section{REFERENCES}

[1] H. Bray, The Penrose inequality in general relativity and volume comparison theorems involving scalar curvature, $\mathrm{PhD}$ thesis, Stanford University (1997)

[2] H. Bray, Proof of the Riemannian Penrose inequality using the positive mass theorem, J. Diff. Geom. 59, 177-267 (2001)

[3] H. Bray and P. Miao, On the capacity of surfaces in manifolds with nonnegative scalar curvature, Invent. Math. 172, 459-475 (2008)

[4] S. Brendle, Rigidity phenomena involving scalar curvature, Surveys in Differential Geometry, volume XVII, 179-202 (2012)

[5] S. Brendle, Constant mean curvature surfaces in warped product manifolds, Publ. Math. IHÉS 117, 247-269 (2013)

[6] S. Brendle, P.-K. Hung, and M.-T. Wang, A Minkowski-type inequality for hypersurfaces in the Anti-deSitter-Schwarzschild manifold, arXiv:1209.0669

[7] J. Corvino, A. Gerek, M. Greenberg, and B. Krummel, On isoperimetric surfaces in general relativity, Pacific J. Math. 231, 63-84 (2007)

[8] G. Huisken and T. Ilmanen, The inverse mean curvature flow and the Riemannian Penrose inequality, J. Diff. Geom. 59, 353-437 (2001)

[9] M. Llarull, Sharp estimates and the Dirac operator, Math. Ann. 310, 55-71 (1998)

[10] A. Neves, Insufficient convergence of inverse mean curvature flow on asymptotically hyperbolic manifolds, J. Diff. Geom. 84, 191-229 (2010)

[11] X. Wang, The mass of asymptotically hyperbolic manifolds, J. Diff. Geom. 57, 273-299 (2001)

Department of Mathematics, Stanford University, 450 Serra Mall, Bldg 380, StANFORD, CA 94305 